Artigo Original

Original Article

Marilia Rodrigues Freitas de Souza

Ellen Osborn ${ }^{1}$

Daniela Gil ${ }^{2}$

Maria Cecília Martinelli lório²

Descritores

Perda auditiva

Auxiliares de audição

Reabilitação de deficientes auditivos

Questionários

Criança

Keywords

Hearing loss

Hearing aids

Rehabilitation of hearing impaired

Questionnaires

Child

Endereço para correspondência: Marilia Rodrigues Freitas de Souza R. Botucatu, 802, Vila Clementino, São Paulo (SP), Brasil, CEP: 04023-062.

E-mail: marilia_mari@yahoo.com.br

Recebido em: 7/2/2011

Aceito em: 6/6/2011

\section{Tradução e adaptação do questionário ABEL - Auditory Behavior in Everyday Life para o Português Brasileiro}

\author{
Translation and adaptation of the ABEL - Auditory Behavior \\ in Everyday Life questionnaire into Brazilian Portuguese
}

Objetivo: Traduzir e adaptar o questionário ABEL - Auditory Behavior in Everyday Life - para o Português Brasileiro e estabelecer o perfil do comportamento auditivo de crianças usuárias de aparelhos de amplificação sonora individual (AASI) por meio de sua aplicação. Métodos: Foi realizada a tradução do questionário ABEL, seguida pela retrotradução. Esta versão foi comparada à original em relação à equivalência semântica, sendo gerada uma nova versão em Português. A partir disso, foram entrevistados 31 pais de crianças adaptadas com AASI. Todos responderam a uma anamnese e à versão em Português do questionário ABEL. Os escores obtidos na aplicação do instrumento foram relacionados às variáveis investigadas na anamnese. Resultados: A análise do escore oral-aural revelou diferenças segundo as variáveis grau de perda auditiva e tempo de uso diário dos AASI, sendo que crianças com menores graus de perda e/ou que fazem uso dos AASI por mais tempo apresentam melhor desempenho. Em relação ao escore total e ao escore de consciência auditiva, houve diferenças segundo o tempo de uso diário dos AASI, indicando melhor desempenho daquelas que fazem uso dos AASI por mais tempo. Conclusão: Há coerência entre as versões geradas nas etapas de tradução do questionário, possibilitando a formulação de sua versão em Português. Crianças com menores graus de perda auditiva e/ou que referem maior tempo de uso diário de AASI têm suas atividades diárias menos comprometidas pela perda auditiva. O questionário ABEL é um instrumento apropriado para detalhar o desenvolvimento dos comportamentos auditivos de crianças usuárias de AASI.

\begin{abstract}
Purpose: To translate and adapt the ABEL - Auditory Behavior in Everyday Life - questionnaire into Brazilian Portuguese, and to establish the profile of auditory behavior in a group of children who use hearing aids. Methods: The ABEL questionnaire was translated and back translated. This version was compared to the original version regarding semantic equivalence, generating a new version in Portuguese. After that, 31 parents of children fitted with hearing aids answered an anamnesis and the Portuguese version of the ABEL questionnaire. The scores obtained were related to the variables investigated in the anamnesis. Results: Differences were found in the analysis of the aural-oral score for the variables degree of hearing loss and time of daily use of the hearing aid: children with milder degrees of hearing loss and/or children who use the hearing aid for longer periods showed better performances. There were also differences in total and auditory awareness scores for the variable time of daily use of the hearing aid, indicating that children who user their hearing aids for longer periods daily showed better performances. Conclusion: There was consistency between the versions generated during the translation of the questionnaire, allowing the formulation of its final version in Portuguese. Children with lower degrees of hearing loss and/or who use their hearing aids for longer periods have their daily activities less affected by the hearing loss. The ABEL questionnaire is an appropriate instrument to detail the development of auditory behaviors in children who use hearing aids.
\end{abstract}

Trabalho realizado no Departamento de Fonoaudiologia, Universidade Federal de São Paulo - UNIFESP - São Paulo (SP), Brasil.

(1) Programa de Pós-Graduação (Doutorado) em Distúrbios da Comunicação Humana, Departamento de Fonoaudiologia, Universidade Federal de São Paulo - UNIFESP - São Paulo (SP), Brasil.

(2) Departamento de Fonoaudiologia, Universidade Federal de São Paulo - UNIFESP - São Paulo (SP), Brasil. 


\section{INTRODUÇÃO}

A importância de se investigar como o Sistema Auditivo recebe, analisa e organiza as informações acústicas do ambiente em crianças se deve ao fato de que alterações decorrentes de perdas auditivas na infância restringem a experiência no início da vida. Tal restrição altera o desenvolvimento auditivo e de linguagem e interfere no desenvolvimento mental, social e educacional da criança ${ }^{(1)}$. O diagnóstico da perda auditiva é essencial para estabelecer a conduta de reabilitação, seja ela iniciada com a seleção e adaptação de aparelhos de amplificação sonora individual (AASI) ou com a opção pelo implante coclear.

Para qualquer que seja a escolha, é necessária uma medida do benefício resultante da intervenção. Na população adulta, geralmente faz-se uso de instrumentos padronizados que permitem a avaliação do benefício com uso da amplificação e sua comparação com dados obtidos na literatura e dados de outros pacientes. No Brasil, a utilização desses instrumentos para crianças não é difundida. Além disso, sua elaboração é dificultada pela variação das necessidades auditivas das crianças com a passagem do tempo, bem como pelas diferenças geradas pelos distintos graus e configurações de perda ${ }^{(2)}$.

Em outros países, a aplicação de questionários aos pais e cuidadores para monitorar o progresso das habilidades auditivas e verbais da criança já é uma prática comum. Um exemplo de questionário deste tipo é o Auditory Behavior in Everyday Life - $\mathrm{ABEL}^{(3)}$.

Sabendo das vantagens que o uso desse tipo de instrumento proporciona no processo de adaptação de dispositivos auditivos eletrônicos na população infantil, foram objetivos deste trabalho traduzir e adaptar o questionário ABEL - Auditory Behavior in Everyday Life para o Português Brasileiro e estabelecer o perfil do comportamento auditivo de crianças usuárias de aparelhos de amplificação sonora individual (AASI) por meio de sua aplicação .

\section{MÉTODOS}

Trata-se de estudo transversal, descritivo, realizado no período compreendido entre junho de 2008 e maio de 2009 , aprovado pelo Comitê de Ética em Pesquisa da Universidade Federal de São Paulo - UNIFESP, sob o protocolo de número 1097/08. Participaram da pesquisa pais e/ou cuidadores previamente esclarecidos sobre os objetivos e métodos do estudo, que autorizaram o uso dos dados coletados, assinando o Termo de Consentimento Livre e Esclarecido (TCLE).

Inicialmente, o questionário $\mathrm{ABEL}^{(3)}$ foi traduzido e adaptado para a língua portuguesa. Esse instrumento tem como objetivo investigar o comportamento auditivo da criança de quatro a 14 anos de idade, deficiente auditiva, em seu dia-a-dia.

O ABEL é composto por 24 itens, cuja soma da pontuação resulta no escore total. As questões são ainda divididas em três fatores: 11 referentes ao aspecto aural-oral, que verifica a recepção auditiva e a resposta verbal aos sons; dez referentes à consciência auditiva e aos sons ambientais e cinco referentes às habilidades sociais, de conversação e de independência fun- cional. Duas das 24 questões (questões 18 e 22) estão presentes em dois fatores (oral-aural e de consciência auditiva). O tempo médio para aplicação deste questionário é de aproximadamente 20 minutos.

A etapa de tradução e adaptação cultural foi realizada da seguinte forma: dois tradutores brasileiros independentes, que conheciam os propósitos do estudo, efetuaram a tradução do instrumento, do Inglês para o Português. As duas versões geraram uma versão única de tradução, que foi submetida à retrotradução (do Português para o Inglês) por uma fonoaudióloga que não conhecia o instrumento original. Esta versão foi submetida a dois profissionais, fonoaudiólogos, com conhecimento do Inglês, para comparar as versões (original e retrotraduzida), em relação à equivalência semântica/idiomática. O objetivo foi garantir a tradução correta, sendo gerada assim uma nova versão em Português do questionário.

A partir disto, foram entrevistados 31 pais e/ou cuidadores de crianças de 4 anos e 6 meses a 13 anos e 6 meses, atendidas no Núcleo Integrado de Assistência, Pesquisa e Ensino em Audição - NIAPEA da UNIFESP. As crianças deveriam obedecer aos seguintes critérios de elegibilidade: apresentar perda auditiva de tipo neurossensorial, mista ou condutiva de grau leve a profundo e fazer uso de AASI bilateralmente. Foram excluídas as crianças com problemas evidentes de saúde que impedissem a observação das atividades referidas no questionário, tais como retardo mental e/ou outros comprometimentos neurológicos graves.

Inicialmente, foi realizada uma anamnese com os 31 responsáveis, (compostos por um tio, três avós, três pais e 24 mães dos pacientes) formulada pelas próprias pesquisadoras, com base no roteiro de entrevista empregado no Ambulatório de Avaliação Audiológica Infantil da UNIFESP. Nesta entrevista, procurou-se investigar dados pessoais, dados referentes à perda auditiva (início, duração, fatores etiológicos, histórico familiar), época da intervenção, tempo de uso diário dos AASI, dados escolares e de saúde geral. Foi realizada também a consulta ao prontuário de cada paciente, a fim de detalhar o tipo e grau da perda auditiva, sua localização (uni ou bilateral), sua característica (estável, progressiva ou flutuante), o modelo e a marca dos AASI e o tipo de molde utilizado.

Após a entrevista, foi aplicado oralmente o questionário ABEL traduzido e adaptado para o Português. Para cada item, os entrevistados deveriam optar por uma das seguintes respostas, com relação à frequência do comportamento referido, apresentado pela criança: nunca (0 ponto), quase nunca (1 ponto), ocasionalmente ( 2 pontos), cerca de metade do tempo ( 3 pontos), frequentemente ( 4 pontos), quase sempre ( 5 pontos) e sempre (6 pontos). Quanto maior a pontuação obtida, melhor o desempenho da criança para cada um dos aspectos medidos pelo questionário, com exceção das questões 11 e 20, cujas pontuações são inversas.

Ao final, foi realizada a análise estatística dos dados coletados. As pontuações obtidas foram relacionadas às diferentes variáveis pesquisadas na entrevista e nos prontuários, a saber: grau de perda auditiva, tempo de uso diário dos AASI, realização ou não de terapia, tipo de abordagem empregada na mesma e tipo de escola frequentada pela criança. 
As variáveis qualitativas foram representadas por frequência absoluta (n) e relativa (\%), e as quantitativas por média, mediana, desvio-padrão, $1^{\circ}$ quartil, $3^{\circ}$ quartil e intervalo de confiança. $\mathrm{Na}$ análise dos dados coletados em anamnese e na aplicação do questionário foram empregados os seguintes testes não paramétricos: teste de Igualdade de Duas Proporções, teste de Kruskal-Wallis e teste de Mann-Whitney.

O nível de significância adotado foi de 0,05 (5\%) e todos os intervalos de confiança apresentados ao longo do trabalho foram construídos com $95 \%$ de confiança estatística.

\section{RESULTADOS}

Tradução e adaptação do questionário ABEL - Auditory Behavior in Everyday Life - para o Português Brasileiro

As versões original, traduzida e retrotraduzida do questionário ABEL - Auditory Behavior in Everyday Life - foram comparadas e apresentaram coerência, o que possibilitou obter a versão final traduzida do instrumento (Figura 1).

\section{Auditory Behavior in Everyday Life (ABEL)}

Comportamento Auditivo nas Atividades Diárias (CAAD)

Nome da criança:

Preenchido por:

Data:

Instruções: Gostaríamos de saber como você observa o desenvolvimento auditivo de sua criança. Por favor, circule o número ao lado de cada item que melhor descreve o comportamento da criança durante a última semana.
0 Nunca
1 Quase nunca
2 Ocasionalmente
3 Cerca de metade do tempo
4 Frequentemente
5 Quase sempre
6 Sempre

1. Inicia conversas com pessoas familiares.

2. Chama uma pessoa pelo nome para chamar sua atenção.

3. Diz "por favor" e "obrigado" sem ser lembrado.

4. Responde verbalmente ao cumprimentar familiares.

5. Inicia conversas com pessoas não familiares.

6. Respeita troca de turnos na conversação.

7. Atende ao telefone adequadamente.

8. Atende ao chamado de seu nome estando no mesmo ambiente.

9. Conversa utilizando uma intensidade normal de voz.

10. Solicita ajuda em situações necessárias.

11. Produz sons vocais inapropriados.

12. Demonstra interesse nas conversas que ocorrem em torno dele / dela.

13. Responde verbalmente ao cumprimento de pessoas não familiares.

14. Diz os nomes dos irmãos, membros da família e colegas de classe.

15. Atende a uma batida na porta ou campainha.

16. Sussura uma mensagem pessoal.

17. Fica em silêncio quando solicitado.

18. Pergunta sobre sons que escuta a sua volta (ex: aviões, caminhões e animais).

19. Reconhece quando produz sons intensos (por exemplo, batidas de porta, batidas de pé).

20. Ignora o toque do telefone.

21. Brinca cooperativamente em um pequeno grupo sem supervisão de adultos.

22. Canta.

23. Sabe quando as próteses auditivas não estão funcionando.

24. Faz experiências com sons recém descobertos.

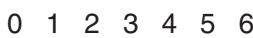

$\begin{array}{lllllll}0 & 1 & 2 & 3 & 4 & 5 & 6\end{array}$

$\begin{array}{lllllll}0 & 1 & 2 & 3 & 4 & 5 & 6\end{array}$

$\begin{array}{lllllll}0 & 1 & 2 & 3 & 4 & 5 & 6\end{array}$

$\begin{array}{lllllll}0 & 1 & 2 & 3 & 4 & 5 & 6\end{array}$

$\begin{array}{lllllll}0 & 1 & 2 & 3 & 4 & 5 & 6\end{array}$

$\begin{array}{lllllll}0 & 1 & 2 & 3 & 4 & 5 & 6\end{array}$

$\begin{array}{lllllll}0 & 1 & 2 & 3 & 4 & 5 & 6\end{array}$

$\begin{array}{lllllll}0 & 1 & 2 & 3 & 4 & 5 & 6\end{array}$

$\begin{array}{lllllll}0 & 1 & 2 & 3 & 4 & 5 & 6\end{array}$

$\begin{array}{lllllll}0 & 1 & 2 & 3 & 4 & 5 & 6\end{array}$

$\begin{array}{lllllll}0 & 1 & 2 & 3 & 4 & 5 & 6\end{array}$

$\begin{array}{lllllll}0 & 1 & 2 & 3 & 4 & 5 & 6\end{array}$

$\begin{array}{lllllll}0 & 1 & 2 & 3 & 4 & 5 & 6\end{array}$

$\begin{array}{lllllll}0 & 1 & 2 & 3 & 4 & 5 & 6\end{array}$

$\begin{array}{lllllll}0 & 1 & 2 & 3 & 4 & 5 & 6\end{array}$

$\begin{array}{lllllll}0 & 1 & 2 & 3 & 4 & 5 & 6\end{array}$

$\begin{array}{lllllll}0 & 1 & 2 & 3 & 4 & 5 & 6\end{array}$

$\begin{array}{lllllll}0 & 1 & 2 & 3 & 4 & 5 & 6\end{array}$

$\begin{array}{lllllll}0 & 1 & 2 & 3 & 4 & 5 & 6\end{array}$

$\begin{array}{lllllll}0 & 1 & 2 & 3 & 4 & 5 & 6\end{array}$

$\begin{array}{lllllll}0 & 1 & 2 & 3 & 4 & 5 & 6\end{array}$

$\begin{array}{lllllll}0 & 1 & 2 & 3 & 4 & 5 & 6\end{array}$

$\begin{array}{lllllll}0 & 1 & 2 & 3 & 4 & 5 & 6\end{array}$

\footnotetext{
INSTRUÇÕES PARA A PONTUAÇÃO (para uso da clínica):

Escores reversos para as questões 11 e 20.

Some as respostas e divida por 24 para obter o escore total

Oral-aural Fator $1=(1+2+3+4+10+12+13+14+18+21+22) / 11$

Consciência auditiva Fator $2=(7+8+15+16+18+19+20+22+23+24) / 10$

Habilidades Sociais/de Conversação Fator $3=(5+6+9+11+17) / 5$
}

Figura 1. Versão do questionário ABEL - Auditory Behavior in Everyday Life, traduzida e adaptada para o Português Purdy SC, Farrington DR, Moran CA, Chard LL, Hodgson SA. A parental questionnaire to evaluate children's Auditory Behavior in Everyday Life (ABEL). Am J Audiol. 2002;11(2):72-82 [por] Marília Rodrigues Freitas de Souza, Daniela Gil, Hellen Osborn, Maria Cecília Martinelli lorio 


\section{Aplicação do questionário ABEL - Auditory Behavior in Everyday Life/CAAD - Comportamento Auditivo nas Atividades Diárias}

Procurou-se detalhar a amostra quanto ao gênero. Foram avaliados $13(41,9 \%)$ pacientes do gênero feminino e 18 $(58,1 \%)$ pacientes do gênero masculino. Por meio do teste de Igualdade de duas Proporções foi possível verificar que não houve diferença na distribuição entre os gêneros $(\mathrm{p}=0,204)$.

Foram analisadas as variáveis quantitativas referentes à idade e escore do questionário (total, oral-aural, de consciência auditiva e de habilidades sociais e conversação). Os pacientes apresentaram média de idade de 104,3 meses $\pm 33,3$. Quanto aos escores, apresentaram pontuação média de $3,87 \pm 1,24$ para o escore total; $4,10 \pm 1,23$ para o escore oral-aural; 4,04 $\pm 1,52$ para o escore de consciência auditiva e $3,03 \pm 1,42$ para o escore de habilidades sociais e de conversação.

A amostra foi distribuída conforme o grau de perda auditiva apresentado pelas crianças. Dos participantes, nove $(29,0 \%)$ apresentaram perda auditiva de grau leve ou moderado, dez $(32,3 \%)$ apresentaram perda auditiva de grau moderadamente severo ou severo e $12(38,7 \%)$ apresentaram perda auditiva de grau profundo. Foi realizada uma análise com o objetivo de comparar o escore total e cada um dos três escores específicos segundo o grau de perda auditiva, apresentado pelas crianças (Tabela 1).

É possível verificar que houve diferença para escore oral-aural, sendo que crianças com menores graus de perda auditiva apresentaram melhor desempenho no questionário.

A amostra foi dividida em dois grupos, conforme o tempo de uso diário dos AASI referido pelos pais. Dos participantes, dez $(32,2 \%)$ faziam uso dos AASI por período inferior a $10 \mathrm{~h} /$ dia e $21(67,7 \%)$ faziam uso dos AASI por tempo igual ou superior a $10 \mathrm{~h} /$ dia. Foi realizada uma análise com o objetivo de comparar o escore total e cada um dos três escores específicos com o tempo de uso diário dos AASI pelas crianças (Tabela 2).

É possível verificar que houve diferença ao comparar o tempo de uso dos AASI com as pontuações obtidas nos escores total, oral-aural e de consciência auditiva. Nota-se que as crianças que fazem uso dos AASI por maior tempo diário apresentaram maiores pontuações.

Na sequência, a amostra foi distribuída em dois grupos, de acordo com a realização ou não de terapia fonoaudiológica (atual ou anterior). Dos pacientes, cinco $(16,1 \%)$ nunca reali-

Tabela 1. Medidas descritivas e comparação dos escores obtidos de acordo com o grau da perda auditiva

\begin{tabular}{|c|c|c|c|c|c|c|c|c|c|}
\hline & Grau & Média & Mediana & DP & Q1 & Q3 & $\mathrm{n}$ & IC & Valor de $p$ \\
\hline & Leve/Mod & 4,52 & 4,5 & 0,47 & 4,2 & 4,8 & 9 & 0,31 & \\
\hline \multirow[t]{2}{*}{ Escore total } & Mod Sev/Severo & 4,14 & 4,3 & 0,71 & 3,7 & 4,5 & 10 & 0,44 & $0,082^{\#}$ \\
\hline & Leve/Mod & 4,88 & 4,8 & 0,35 & 4,7 & 5,0 & 9 & 0,23 & \\
\hline \multirow[t]{2}{*}{ Oral-aural } & Mod Sev/Severo & 4,31 & 4,3 & 0,89 & 3,8 & 4,8 & 10 & 0,55 & $0,021^{*}$ \\
\hline & Leve/Mod & 4,67 & 4,8 & 0,56 & 4,4 & 5,1 & 9 & 0,37 & \\
\hline \multirow[t]{3}{*}{ Consciência auditiva } & Mod Sev/Severo & 4,44 & 4,4 & 1,00 & 3,8 & 5,3 & 10 & 0,62 & 0,219 \\
\hline & Profundo & 3,24 & 3,6 & 2,03 & 1,5 & 4,7 & 12 & 1,15 & \\
\hline & Leve/Mod & 3,40 & 3,8 & 1,48 & 2,8 & 4,6 & 9 & 0,97 & \\
\hline Habilidades sociais/conversação & Mod Sev/Severo & 3,06 & 3,0 & 1,12 & 2,5 & 3,6 & 10 & 0,69 & 0,559 \\
\hline
\end{tabular}

* Valores significativos ( $\mathrm{p} \leq 0,05)$ - Teste Kruskal-Wallis

* Valores próximos ao nível de significância $(p \leq 0,05)$ - Teste Kruskal-Wallis

Legenda: Mod = moderado; Mod Sev = moderadamente severo; Q1 = $1^{\circ}$ quartil; Q3 = $3^{\circ}$ quartil; IC = intervalo de confiança; DP = desvio-padrão

Tabela 2. Medidas descritivas e comparação dos escores obtidos de acordo com o tempo de uso diário do AASI

\begin{tabular}{|c|c|c|c|c|c|c|c|c|c|}
\hline & Tempo de uso & Média & Mediana & DP & Q1 & Q3 & $\mathrm{n}$ & IC & Valor de $p$ \\
\hline \multirow{2}{*}{ Escore total } & $<10 \mathrm{~h} /$ dia & 3,31 & 3,5 & 1,19 & 2,7 & 4,2 & 10 & 0,74 & \multirow{2}{*}{$0,021^{*}$} \\
\hline & $\geq 10 \mathrm{~h} / \mathrm{dia}$ & 4,14 & 4,4 & 1,20 & 3,8 & 4,9 & 21 & 0,51 & \\
\hline \multirow{2}{*}{ Oral-aural } & $<10 \mathrm{~h} /$ dia & 3,51 & 3,7 & 1,22 & 2,7 & 4,5 & 10 & 0,75 & \multirow{2}{*}{$0,045^{\star}$} \\
\hline & $\geq 10 \mathrm{~h} / \mathrm{dia}$ & 4,38 & 4,7 & 1,16 & 3,8 & 5,3 & 21 & 0,49 & \\
\hline \multirow{2}{*}{ Consciência auditiva } & $<10 \mathrm{~h} / \mathrm{dia}$ & 3,36 & 3,8 & 1,41 & 2,9 & 4,5 & 10 & 0,88 & \multirow{2}{*}{$0,033^{*}$} \\
\hline & $\geq 10 \mathrm{~h} / \mathrm{dia}$ & 4,37 & 4,8 & 1,49 & 3,8 & 5,4 & 21 & 0,64 & \\
\hline \multirow{2}{*}{ Habilidades sociais/conversação } & $<10 \mathrm{~h} /$ dia & 2,58 & 2,8 & 1,33 & 1,9 & 3,3 & 10 & 0,83 & \multirow{2}{*}{0,196} \\
\hline & $\geq 10 \mathrm{~h} / \mathrm{dia}$ & 3,24 & 3,6 & 1,44 & 2,4 & 4,6 & 21 & 0,62 & \\
\hline
\end{tabular}

Valores significativos $(p \leq 0,05)$ - Teste de Mann-Whitney

Legenda: $\mathrm{Q} 1=1^{\circ}$ quartil; $\mathrm{Q} 3=3^{\circ}$ quartil; IC = intervalo de confiança; $\mathrm{DP}=$ desvio-padrão 
zaram terapia e $26(83,9 \%)$ já realizaram ou realizam terapia fonoaudiológica. Foi feita uma análise com o objetivo de comparar o escore total e cada um dos três escores específicos entre os grupos(Tabela 3).

As 26 crianças $(83,9 \%$ do total) que realizaram ou realizam terapia fonoaudiológica foram distribuídas em dois grupos, de acordo com a abordagem terapêutica utilizada. Destas, $18(69,2 \%)$ frequentaram terapia com abordagem aurioral e oito $(30,8 \%)$ frequentaram terapia com abordagens distintas (LIBRAS, verbo tonal, comunicação total). Foi realizada a comparação entre os escores obtidos (total e de cada um dos três escores específicos) nestes grupos (Tabela 4).
Verificou-se que não houve diferença ao comparar a abordagem empregada em terapia e os diferentes escores.

Por fim, a amostra foi dividida em dois grupos, de acordo com o tipo de escola frequentada pelas crianças. Uma das crianças não ia à escola e foi excluída dessa análise. Dos participantes, seis $(20,0 \%)$ frequentavam escola especial para deficientes auditivos e $24(80,0 \%)$ frequentavam escola regular. Os escores (total e cada um dos três escores específicos) foram comparados ao tipo de escola cursada (Tabela 5).

Não houve diferença ao comparar o tipo de escola e os diferentes escores.

Tabela 3. Medidas descritivas e comparação dos escores obtidos de acordo com a realização de terapia fonoaudiológica

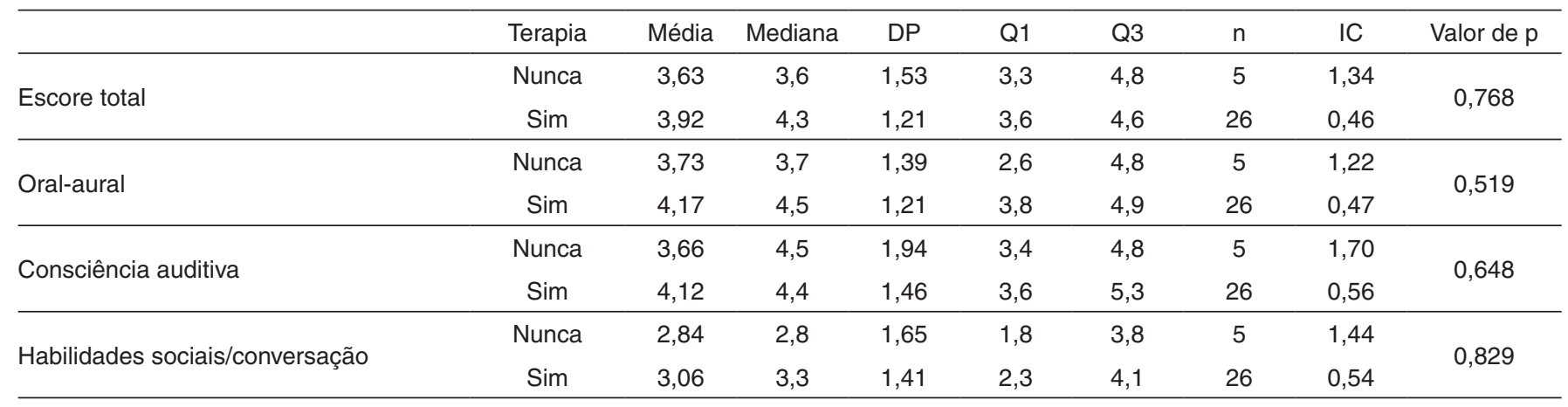

Teste de Mann-Whitney $(p \leq 0,05)$

Legenda: $\mathrm{Q} 1=1^{\circ}$ quartil; $\mathrm{Q} 3=3^{\circ}$ quartil; IC = intervalo de confiança; $\mathrm{DP}=$ desvio-padrão

Tabela 4. Medidas descritivas e comparação dos escores obtidos de acordo com a abordagem empregada em terapia

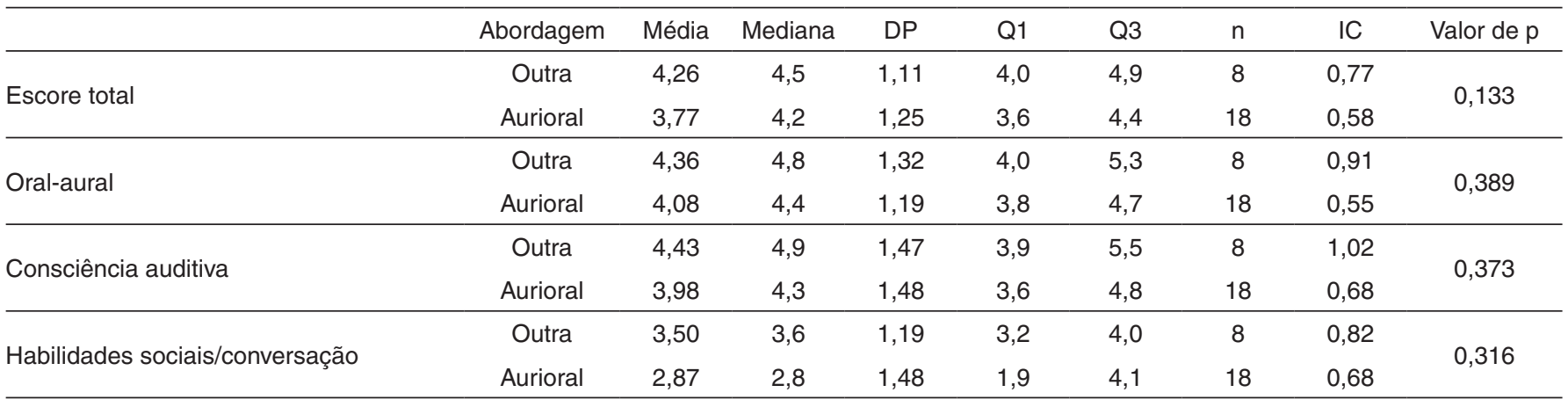

Teste de Mann-Whitney $(\mathrm{p} \leq 0,05)$

Legenda: $\mathrm{Q} 1=1^{\circ}$ quartil; $\mathrm{Q} 3=3^{\circ}$ quartil; IC = intervalo de confiança; $\mathrm{DP}=$ desvio-padrão

Tabela 5. Medidas descritivas e comparação dos escores obtidos de acordo com o tipo de escola frequentada pela criança

\begin{tabular}{|c|c|c|c|c|c|c|c|c|c|}
\hline & Tipo de escola & Média & Mediana & DP & Q1 & Q3 & $\mathrm{n}$ & IC & Valor de $p$ \\
\hline \multirow{2}{*}{ Escore total } & Especial & 3,45 & 4,3 & 2,16 & 1,7 & 5,0 & 6 & 1,73 & \multirow{2}{*}{0,959} \\
\hline & Regular & 4,09 & 4,3 & 0,78 & 3,6 & 4,6 & 24 & 0,31 & \\
\hline \multirow{2}{*}{ Oral-aural } & Especial & 3,83 & 4,5 & 1,99 & 2,3 & 5,3 & 6 & 1,59 & \multirow{2}{*}{0,979} \\
\hline & Regular & 4,25 & 4,5 & 0,94 & 3,8 & 4,8 & 24 & 0,38 & \\
\hline \multirow{2}{*}{ Consciência auditiva } & Especial & 3,37 & 4,1 & 2,29 & 1,5 & 5,2 & 6 & 1,83 & \multirow{2}{*}{0,516} \\
\hline & Regular & 4,36 & 4,5 & 1,04 & 3,8 & 5,1 & 24 & 0,42 & \\
\hline \multirow{2}{*}{ Habilidades sociais/conversação } & Especial & 2,97 & 3,9 & 2,11 & 1,4 & 4,5 & 6 & 1,69 & \multirow{2}{*}{0,835} \\
\hline & Regular & 3,13 & 3,0 & 1,20 & 2,4 & 3,8 & 24 & 0,48 & \\
\hline
\end{tabular}

Teste de Mann-Whitney $(\mathrm{p} \leq 0,05)$

Legenda: $\mathrm{Q} 1=1^{\circ}$ quartil; $\mathrm{Q} 3=3^{\circ}$ quartil; IC = intervalo de confiança; $\mathrm{DP}=$ desvio-padrão 


\section{DISCUSSÃO}

\section{Tradução e adaptação do questionário ABEL - Auditory Behavior in Everyday Life - para o Português Brasileiro}

Quanto à tradução e adaptação do questionário para a língua portuguesa, foi observada coerência entre as versões original, retrotraduzida e final do questionário. Poucas dificuldades foram encontradas. A maior delas foi referente à tradução da questão 16: Will whisper a personal message, em que, de acordo com o Dicionário Michaelis de Inglês-Português, whisper significa cochicho, murmúrio, sussurro, e personal message uma mensagem pessoal. Assim, o item ficou traduzido ao final como "sussurra uma mensagem pessoal". Apesar disso, o item não foi bem compreendido pelos pais e, como o questionário foi aplicado oralmente, foram possíveis explicações e substituições pelo sinônimo "cochichar".

Outros itens que trouxeram dúvidas de compreensão e interpretação, não pela tradução, mas pelo vocabulário pouco usual, foram os itens 6 ("respeita troca de turnos na conversação"), 11 ("produz sons vocais inapropriados") e 24 ("faz experiências com sons recém descobertos"). Tais questões necessitaram, por vezes, da substituição de termos por sinônimos e explicações de significado.

Embora seja um questionário aparentemente simples e objetivo, o fato de possuir, para cada uma das questões, sete opções de respostas, dificulta sua aplicação. Para esclarecer o que seria a graduação de frequência de ocorrência, foi explicado aos participantes que o intuito era que pontuassem quanto cada um dos comportamentos apontados no questionário ocorria em uma semana. Para que não se esquecessem das opções de respostas que tinham, foi oferecida uma prancha, em papel cartão, contendo as sete opções. As perguntas eram feitas oralmente, mas o acesso às respostas poderia ser recordado durante toda aplicação do instrumento.

\section{Aplicação do questionário ABEL - Auditory Behavior in Everyday Life/Comportamento Auditivo nas Atividades Diárias (CAAD)}

Os dados resultantes da aplicação do questionário ABEL/ CAAD mostraram as seguintes médias de escores: total de 3,87 pontos; oral-aural de 4,10 pontos; de consciência auditiva de 4,04 pontos; e de habilidades sociais e de conversação de 3,03 pontos. Assim, comparando os quatro escores, as crianças deficientes auditivas apresentaram piores pontuações para as habilidades sociais. Este fato se deve à privação da linguagem, ao desencorajamento da independência e da responsabilidade, e à ausência de aprendizado incidental ${ }^{(4)}$. A criança precisa de modelos para aprender sobre os comportamentos aceitos socialmente, mas a dificuldade na comunicação de deficientes auditivos impede que eles recebam explicações precisas, coerentes e completas para desenvolver a percepção do que lhes é exigido.

Ao relacionar o desempenho das crianças no questionário com o grau da deficiência auditiva apresentada por elas, foram encontradas diferenças no escore oral-aural, para o qual crianças com menores graus de perda auditiva apresentaram melhor desempenho.

Durante a aplicação do questionário ABEL em sua primeira versão ${ }^{(3)}$, ainda com 49 itens, foram observados piores desempenhos das crianças com perda auditiva de grau severo a profundo quando comparadas às crianças com perdas auditivas de grau leve a moderado, dado similar ao obtido no presente estudo. Pelo fato da pesquisa citada apresentar outros objetivos (análise da confiabilidade do instrumento), não houve o detalhamento da pontuação obtida para cada um dos escores. $\mathrm{Na}$ literatura, não foram encontrados outros estudos com o questionário ABEL que tenham realizado comparação similar. Foram encontradas pesquisas que utilizaram outras escalas para comparar a pontuação obtida por crianças ouvintes e crianças com deficiência auditiva, que constataram que estas últimas apresentaram desempenhos inferiores em medidas subjetivas do comportamento auditivo e de linguagem ${ }^{(5-8)}$. Outros estudos apontaram ainda para a diferença na linguagem expressiva também medida por meio de instrumentos subjetivos, dependendo do grau de perda auditiva apresentado pelas crianças, e verificaram melhores desempenhos nas crianças com menores graus de $\operatorname{perda}^{(8,9)}$.

Quanto ao tempo de uso diário dos AASI, outras pesquisas também consideraram este fator no desenvolvimento de habilidades auditivas nas crianças com algum grau de perda auditiva. Um estudo aponta que alguns fatores podem estar relacionados à melhor adaptação aos aparelhos de amplificação sonora individual ${ }^{(10)}$. Segundo estes autores, crianças com perda entre 50 e 90 dBNA usam AASI com mais freqüência. No presente trabalho, foram obtidos achados compatíveis com tal afirmação: das 21 crianças cujos pais relataram tempo de uso de AASI igual ou superior a dez horas por dia, $12(57,1 \%)$ apresentaram perda auditiva de grau moderado a severo - dentro da faixa de 50 a 90 dBNA. Na pesquisa citada, $19 \%$ das crianças não faziam uso constante dos AASI, valor inferior ao encontrado no presente estudo $(32,2 \%)$.

Outra pesquisa também considerou o mesmo fator no desenvolvimento de habilidades auditivas de crianças com algum grau de perda ${ }^{(11)}$. Por meio de um questionário de autoavaliação, os autores verificaram que $63 \%$ dos deficientes auditivos utilizavam os AASI em todos os locais que frequentavam, retirando-as apenas para tomar banho e dormir, valor inferior ao encontrado na presente pesquisa $(67,7 \%)$.

Em investigação realizada com 12 crianças usuárias de AASI foi possível verificar que cinco delas $(41,7 \%)$ utilizavam o AASI por 11 a 15 horas diárias; três $(25,0 \%)$ por 6 a 10 horas; três $(25,0 \%)$ por 1 a 5 horas e uma $(8,3 \%)$, por menos de uma hora $^{(12)}$. O presente estudo mostrou porcentagens maiores em crianças cujos pais ou responsáveis relataram fazer uso de AASI de forma mais constante $(67,7 \%)$.

Também no presente estudo, foi possível verificar diferença ao comparar o tempo de uso diário de aparelhos de amplificação sonora individual com as pontuações obtidas no questionário ABEL/CAAD para os escores total, oral-aural e de consciência auditiva. Verificou-se, em todas as análises, que quem usou AASI por maior tempo diário, apresentou maiores pontuações.

Tais resultados são compatíveis com a ideia de que dispo- 
sitivos de amplificação sonora possibilitam uma melhora na entrada auditiva e, consequentemente, uma melhora do comportamento nas atividades diárias (medido pelo escore total), no fator aural-oral (receber e reagir ao som, fazendo uso da forma oral de comunicação) e no fator de consciência auditiva (diferenciar a presença e a ausência dos sons). Para a melhora das habilidades auditivas, de linguagem e sociais são necessários alguns cuidados, tais como o uso correto dos AASI. Para que estes possam auxiliar o deficiente auditivo, faz-se necessário mantê-los em bom funcionamento e em uso contínuo. Se os aparelhos de amplificação sonora individual não forem usados regularmente, a criança receberá informações interrompidas do mundo sonoro, o que dificultará o desenvolvimento das funções auditivas, de fala e de linguagem ${ }^{(13)}$.

Quanto à realização de terapia fonoaudiológica, é importante ressaltar que tal atividade garante a melhora de todos os aspectos (aural-oral, de consciência auditiva e de habilidades sociais/conversação) que interferem no comportamento auditivo nas atividades diárias (escore total). Porém, a análise da interferência desta variável no comportamento das crianças investigadas não revelou diferenças. Além do questionário ABEL, outros instrumentos podem ser utilizados para avaliar os benefícios alcançados na terapia de crianças com deficiência auditiva, sejam elas usuárias de AASI ou implantes cocleares. Como exemplo, pode-se citar uma pesquisa que verificou a eficácia do uso do implante coclear e da terapia fonoaudiológica em crianças menores de um ano de idade, por meio da aplicação do questionário IT-MAIS - Infant Toddler Meaningful Auditory Integration Scale aos pais das crianças antes da cirurgia, três meses após, seis meses após e a cada seis meses até que se atingisse um nível máximo de resposta. Também foram aplicados testes de recepção e compreensão de fonemas, palavras e sentenças, quando pertinentes. Foi observada melhora substancial para todas as 18 crianças nos resultados do IT-MAIS, comparando as respostas fornecidas no pré-operatório, quando faziam uso de AASI, com a condição após seis meses de cirurgia de implante coclear. Assim, a terapia associada à adaptação do implante influencia na melhora do desempenho medido pelo questionário ${ }^{(14)}$, o que não foi observado no presente estudo, com uso do instrumento traduzido.

Com relação à abordagem empregada em terapia atual ou anterior, não houve diferença entre os diferentes escores analisados segundo esta variável. Na literatura, admite-se que a abordagem empregada em terapia fonoaudiológica de deficientes auditivos deve ser pensada caso a caso, levando-se em consideração variados aspectos, como grau da perda auditiva, época de instalação, etiologia, idade de diagnóstico e de início da reabilitação, aspectos familiares, dentre outros. Desta forma, há uma série de variáveis que podem interferir no melhor aproveitamento de uma determinada abordagem terapêutica por uma criança ${ }^{(15)}$.

A abordagem terapêutica aurioral propiciará o melhor desenvolvimento possível em função, entre outros fatores, do grau de perda auditiva. Quanto menor o grau da perda, melhor será o desempenho da criança com aparelhos de amplificação sonora individual, pois o benefício resultante da amplificação permitirá que o sinal de fala seja recebido de forma adequada ${ }^{(13)}$.
Em crianças com perdas auditivas de graus maiores, mesmo com ganho satisfatório com AASI, muitas vezes o sinal de fala ainda é apresentado de forma insuficiente, o que dificulta o uso exclusivo de estímulos orais de terapia. Nestes casos, é esperado que a criança se beneficie de outras modalidades terapêuticas, tais como sensoriais e gestuais. No presente estudo, para todos os casos em que foi empregado outro tipo de terapia, exceto em uma criança, o grau da perda auditiva diagnosticada na melhor orelha situava-se entre severo e profundo.

Além da questão do grau de perda auditiva, pode-se considerar ainda que os pais, ao iniciarem o processo terapêutico numa abordagem aurioral, podem apresentar uma expectativa elevada de desenvolvimento de fala e linguagem dos filhos. Podem desejar que rapidamente os filhos alcancem metas de vocabulário expressivo e receptivo próximas das de crianças ouvintes, o que nem sempre ocorre e acabam por frustrá-los. Os escores baixos obtidos no instrumento podem ser decorrentes desta frustração.

Não foram encontrados estudos com o questionário empregado na presente pesquisa, nem com outros instrumentos similares, que tivessem como objetivo comparar diferentes propostas de ensino e o comportamento auditivo em atividades diárias.

Não houve diferença ao analisar os diferentes escores em relação ao tipo de escola frequentada pelas crianças. Vale ressaltar que tais achados permitem interrogar até que ponto estão adequados os recursos oferecidos pela atual política educacional inclusiva, promovendo a organização de classes comuns e de serviços de apoio pedagógico especializado ${ }^{(16)}$. Mais do que isso, coloca em questionamento se de fato estão sendo eliminadas as barreiras de comunicação, com garantia aos surdos de acesso à informação e à educação, recordando a Lei $\mathrm{n}^{\circ} 10.098$ de $2000^{(17)}$.

Após o detalhamento e discussão dos achados, pode-se dizer que o questionário $\mathrm{ABEL} / \mathrm{CAAD}$ mostrou-se um instrumento apropriado para avaliar o desenvolvimento dos comportamentos auditivos de crianças usuárias de aparelhos de amplificação sonora individual, fornecendo informações úteis ao fonoaudiólogo que trabalha com reabilitação auditiva.

\section{CONCLUSÃO}

Após a análise crítica dos resultados deste estudo, pode-se concluir que há coerência entre as versões geradas nas distintas etapas de tradução do questionário, possibilitando a formulação da versão final do instrumento na língua portuguesa. Quanto menor o grau de perda auditiva apresentado pelas crianças, melhor é o desempenho medido pelo questionário para o escore oral-aural, ou seja, melhores são as habilidades da criança em receber informações sonoras e responder oralmente às mesmas. Quanto maior o tempo de uso diário dos aparelhos de amplificação sonora individual, melhor é o desempenho medido pelo questionário nos escores total, oral-aural e de consciência auditiva. Assim, o uso contínuo dos aparelhos de amplificação sonora individual contribuí para que a criança obtenha sucesso em suas atividades diárias, estando apta a receber, reter e conscientizar-se das informações sonoras, e comunicar-se na 
dependência de tais capacidades. As habilidades sociais e de conversação não foram influenciadas por nenhum dos fatores pesquisados na história clínica.

\section{REFERÊNCIAS}

1. Azevedo MF. Avaliação audiológica no primeiro ano de vida. In: Lopes Filho OC. Tratado de Fonoaudiologia. São Paulo: Roca; 1997. p. 239-63.

2. Iorio MC, Menegotto IH. Seleção e adaptação de próteses auditivas em crianças. In: Ferreira LP, Befi-Lopes DM, Limongi SC. Tratado de Fonoaudiologia. São Paulo: Roca; 2004. p. 680-93.

3. Purdy SC, Farrington DR, Moran CA, Chard LL, Hodgson SA. A parental questionnaire to evaluate children's Auditory Behavior in Everyday Life (ABEL). Am J Audiol. 2002;11(2):72-82.

4. Domingues AF, Motti TF, Palamin ME. O brincar e as habilidades sociais na interação da criança com deficiência auditiva e mãe ouvinte. Estud Psicol. 2008;25(1):37-44.

5. Kishon-Rabin L, Taitelbaum-Swead R, Ezrati-Vinacour R, Hildesheimer M. Prelexical vocalization in normal hearing and hearing-impaired infants before and after cochlear implantation and Its relation to early auditory skills. Ear Hear. 2005;26(4 Suppl):17S-29S.

6. Wang NY, Eisenberg LS, Johnson KC, Fink NE, Tobey EA, Quittner AL, et al. Tracking development of speech recognition: longitudinal data from hierarchical assessments in the Childhood Development after Cochlear Implantation Study. Otol Neurotol. 2008;29(2):240-5.

7. Rinaldi P, Caselli C. Lexical and grammatical abilities in deaf Italian preschoolers: the role of duration of formal language experience. J Deaf Stud Deaf Educ. 2009;14(1):63-75.
8. Vohr B, Jodoin-Krauzyk J, Tucker R, Johnson MJ, Topol D, Ahlgren M. Early language outcomes of early-identified infants with permanent hearing loss at 12 to 16 months of age. Pediatrics. 2008;122(3):535-44.

9. Pipp-Siegel S, Sedey AL, VanLeeuwen AM, Yoshinaga-Itano C. Mastery Motivation and Expressive Language in young children with hearing loss. J Deaf Stud Deaf Educ. 2003;8(2):133-45.

10. Marttila TI, Karikoski JO. Hearing aid use in Finnish children - impact of hearing loss variables and detection delay. Int J Pediatr Otorhinolaryngol. 2006;70(3):475-80.

11. Boscolo CC, Costa MP, Domingos CM, Perez FC. Avaliação dos benefícios proporcionados pelo AASI em crianças e jovens da faixa etária de 7 a 14 anos. Rev Bras Educ Espec. 2006;12(2):255-68.

12. Couto MI, Lichtig I. Efeitos da amplificação sonora sobre as modalidades comunicativas utilizadas pelos pais. Pró-Fono. 2007;19(1):75-86.

13. Bevilacqua MC, Formigoni GM. Audiologia educacional: uma opção terapêutica para a criança deficiente auditiva. Barueri: Pró-Fono; 1998.

14. Waltzman SB, Roland JT Jr. Cochlear implantation in children younger than 12 months. Pediatrics. 2005;116(4):e487-93.

15. Margall SA, Honora M, Carlovich AL. A reabilitação do deficiente auditivo visando qualidade de vida e inclusão social. Mundo Saúde. 2006;30(1):123-8.

16. Farias SB. As tecnologias da informação e comunicação e a construção do conhecimento pelo aluno surdo [tese]. João Pessoa: Universidade Federal da Paraíba; 2006.

17. Guarinello AC, Berberian AP, Santana AP, Massi G, de Paula M. A inserção do aluno surdo no ensino regular: visão de um grupo de professores do Estado do Paraná. Rev Bras Educ Espec. 2006;12(3):31730. 